\title{
Talc Aspect Ratios Measured by Scanning White Light Interference Microscopy (SWLIM)
}

\author{
R. A. Rose and T. B. Vander Wood \\ *MVA, Inc., 5500 Oakbrook Parkway \#200, Norcross, GA 30093
}

Platy mineral aspect ratios (average plate diameter divided by plate thickness) can play a governing role in behavior in industrial applications $[1,2]$. Conventional methods of measurement include manual techniques such as shadowing [3] or high tilt observation [4] in the scanning electron microscope. Automated measurement of particle diameter combined with measurement of electron scattering loss for measurement of plate thickness has been employed to measure the aspect ratio of platy minerals which are sufficiently thin to be electron transparent [5]. Each of these techniques suffers from some drawback, either in the time required (for manual methods) or the expense of the equipment needed (for automated methods).

We report here a direct method of measuring platy particle aspect ratios using scanning white light interference microscopy (SWLIM). The technique can be applied to any platy particle with a diameter greater than about 3 micrometers. Particle thickness is not a limiting factor, with vertical resolutions below $10 \mathrm{~nm}$ possible.

Samples of commercial talc were dispersed on carefully cleaned glass coverslips and coated with carbon. SWLIM analysis [6] of individual particles was carried out in a Wyko NT-2000 surface profilometer in VSI (vertical scanning interferometry) mode. Particle measurements were obtained at magnifications of 50 or 100x utilizing the "Multiple Region Analysis" option supplied by the manufacturer [7]. For each particle, the $\mathrm{X}$ and $\mathrm{Y}$ caliper dimensions were measured and the mean particle height (thickness) was calculated from a manually determined baseline. The samples were then mounted in the same orientation for imaging and measurement in a JEOL 6500 field emission scanning electron microscope. Individual particles subjected to measurement by SWLIM were located, a secondary electron image of the particle was obtained, and the $\mathrm{X}$ and $\mathrm{Y}$ caliper dimensions measured and recorded. The sample stage was then tilted $60^{\circ}$ away from the detector to present an image of the particle edge. Several measurements of the apparent thickness of the particle were recorded, and the actual particle thickness calculated by correcting the apparent thickness for the $60^{\circ}$ sample tilt.

Figure 1 shows a false-colored contour image of a talc particle, along with a calibration scale. A secondary electron image of the same particle is shown along side. Figure 2 compares the results of measurements of 56 particles by the two techniques. There is excellent agreement in the measure of average diameter, although there is an offset of the intercept of the best fit line of approximately 1 $\mu \mathrm{m}$. There is significantly more scatter in the comparison of measured thickness, which is attributed to the irregular thickness of the particles over their full diameter, see Figure 1. This difference in measured thickness results in scatter in the aspect ratios calculated from the measurements by the two techniques, with the SEM results tending towards higher values. However, for this data set the differences are not significant. The mean aspect ratios measured by SWLIM and SEM are 6.84 and 7.92, respectively. Application of Student's t-test to this data set indicates that there is no difference between these means at the $1 \%$ level of significance. 
References

[1] B. O. Lee et al., J. Mat. Sci., 37 (2002) 1839.

[2] A. Persson Eng. Geol. 50 (1998) p.177.

[3] D. C. Pease, Histological Techniques for Electron Microscopy, Academic Press, Inc., New York, 1964.

[4] M. A. Hayat, Principles and Techniques of Electron Microscopy, Cambridge University Press, Cambridge, 2000.

[5] T.B. Vander Wood et al., Process Mineralogy XIII, R.D. Hagni, ed., The Minerals, Metals \& Materials Society, 1995.

[6] P.C. Caber, Appl. Opt. 32(19) (193) 3438.

[7] WYKO Surface Profilers Technical Reference Manual, 980-085, WYKO Corporation, 1996.
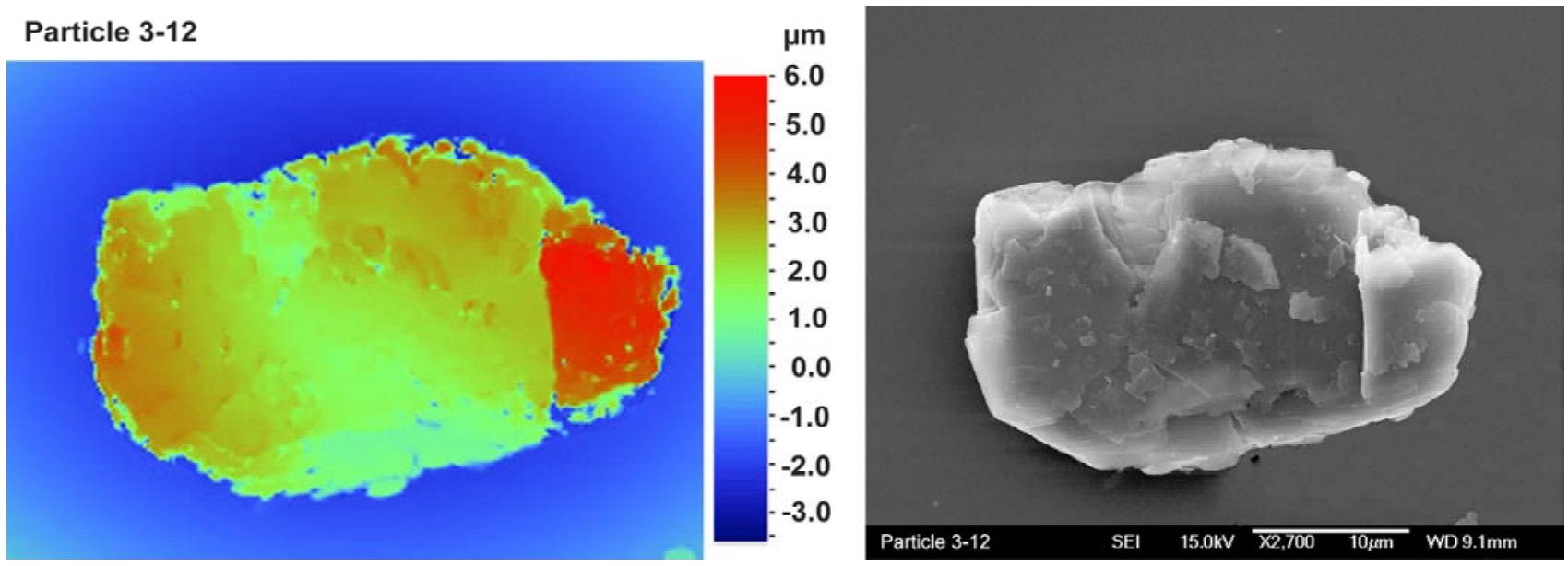

Figure 1. SWLIM false colored image with thickness scale and secondary electron image of the same talc particle. This particle is indicated by an arrow in Figure 2.
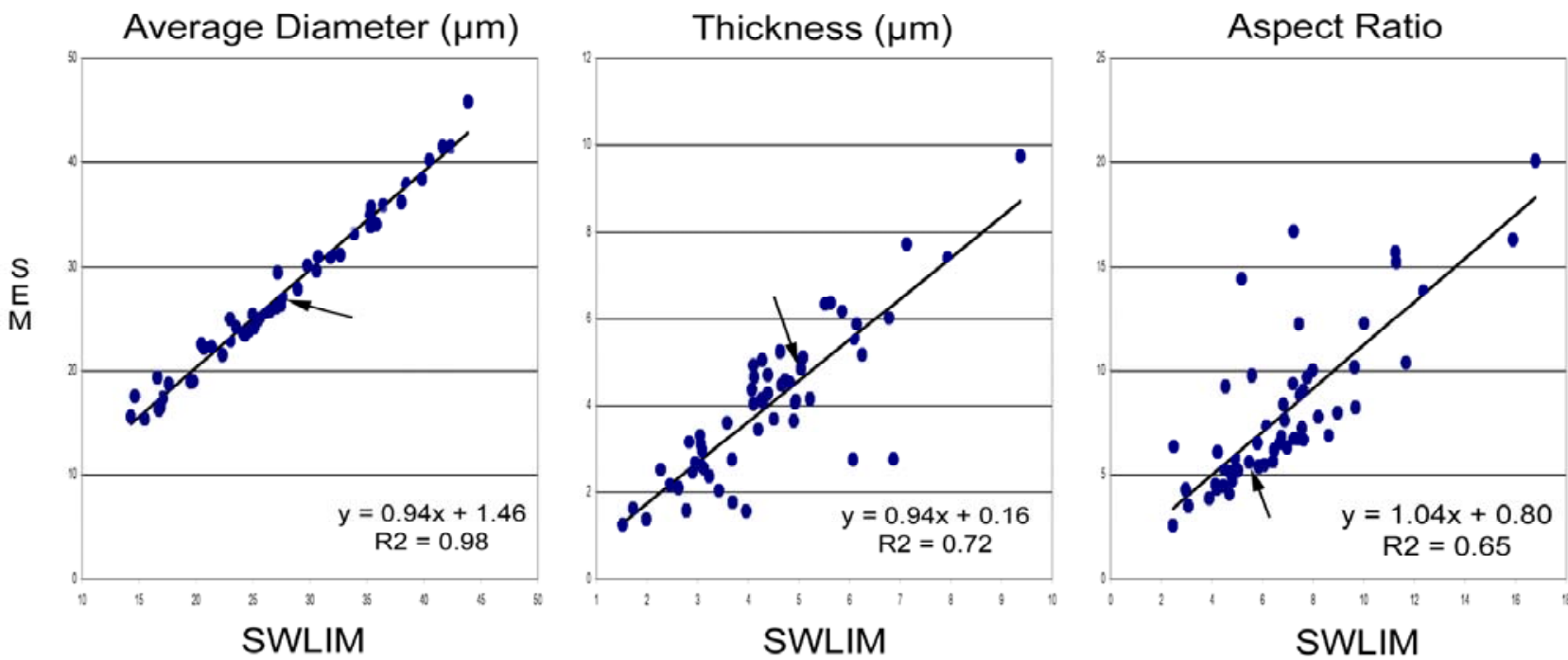

Figure 2. Comparison of aspect ratios determined by FESEM and SWLIM for 56 talc particles 